\title{
Electrochromic Electrochemical Transistors Gated With Polyelectrolyte-Decorated Amyloid Fibrils
}

Deyu Tu, David Nilsson and Robert Forchheimer

\section{Linköping University Post Print}

\section{Tweet}

N.B.: When citing this work, cite the original article.

C2013 IEEE. Personal use of this material is permitted. However, permission to reprint/republish this material for advertising or promotional purposes or for creating new collective works for resale or redistribution to servers or lists, or to reuse any copyrighted component of this work in other works must be obtained from the IEEE.

Deyu Tu, David Nilsson and Robert Forchheimer, Electrochromic Electrochemical Transistors Gated With Polyelectrolyte-Decorated Amyloid Fibrils, 2013, IEEE/OSA Journal of Display Technology, (9), 9, 755-759.

http://dx.doi.org/10.1109/JDT.2013.2278015

Postprint available at: Linköping University Electronic Press

http://urn.kb.se/resolve?urn=urn:nbn:se:liu:diva-99411 


\title{
Electrochromic electrochemical transistors gated with polyelectrolyte-decorated amyloid fibrils
}

\author{
Deyu Tu, David Nilsson, and Robert Forchheimer
}

\begin{abstract}
This paper presents the use of polyelectrolyte-decorated amyloid fibrils as gate electrolyte in electrochromic electrochemical transistors. Conducting polymer alkoxysulfonate poly(3,4-ethylenedioxythiophene) (PEDOT-S) and luminescent conjugate polymer poly(thiophene acetic acid) (PTAA) are utilized to decorate insulin amyloid fibrils for gating lateral poly(3,4-ethylenedioxythiophene):poly(styrene sulfonate) (PEDOT:PSS) electrochemical transistors. In this comparative work, four gate electrolytes are explored, including the polyelectrolytes and their amyloid-fibril complexes. The discrimination of transistor behaviors with different gate electrolytes is understood in terms of an electrochemical mechanism. The combination of luminescent polymers, biomolecules and electrochromic transistors enables multi functions in a single device, for example, the color modulation in monochrome electrochromic display, as well as biological sensing/labeling.
\end{abstract}

Index Terms - amyloid fibrils, biomolecules, electrochemical transistors, PEDOT-S, PTAA

\section{INTRODUCTION}

$\mathrm{O}$ RGANIC semiconductors have been extensively studied for electronic functions in recent years, aiming at low-cost applications [1]. As the most important electrical component in electronics, the transistor has been demonstrated based on a wide variety of organic semiconductors [2]. Most of organic transistors are organic field effect transistors (OFETs), where the conductivity of the semiconductor layer is modulated by field effect doping via an electric field applied across the insulating gate dielectric layer [3]. Another kind of organic transistors named organic electrochemical transistors (OECTs) has been developed in parallel, where the channel current is modulated by electrochemical doping/de-doping via redox mediated by ions from an electrolyte layer [4, 5]. Organic electrochemical transistors are usually operated at low voltage [6] and printable on unconventional substrates (e. g. paper) for large-area applications [7]. Among many applications of electrochemical transistors, such as logic circuits $[6,7]$ and biological sensing [8], electrochromic display is one of the most attractive [9].

Manuscript received February 16, 2012. This work was supported by "OPEN" project at the Center of Organic Electronics (COE) at Linköping University, Sweden, funded by the Strategic Research Foundation SSF.

Deyu Tu and Robert Forchheimer are with the Information Coding, ISY Linköping University, SE-581 83 Linköping Sweden (e-mail: deyu@ isy.liu.se).

David Nilsson is with the Acreo AB, SE-601 17, Norrköping, Sweden.
PEDOT:PSS is a commercially available conducting polymer blend, where its conductivity is dependent on PEDOT's redox state. In presence of ions from electrolytes, PEDOT can be reduced via the following reaction:

$$
\mathrm{PEDOT}^{+} \mathrm{PSS}^{-}+\mathrm{M}^{+}+e^{-} \leftrightarrow \mathrm{PEDOT}^{0}+\mathrm{M}^{+} \mathrm{PSS}^{-}
$$

Driven by bias, the cation $M^{+}$in an electrolyte layer assists the reduction process which reduce the conductivity of PEDOT:PSS. Therefore, PEDOT:PSS is used to be the channel material conducting electrons in organic electrochemical transistors [5-8]. The redox process of PEDOT also changes its optical band gap, resulting in electrochromism [9]. However, it can be used only in monochrome display. To obtain ionic side chains on the conducting PEDOT for biomolecular interaction, a new derivative of PEDOT, alkoxysulfonate PEDOT (PEDOT-S), is used for self-assembly of conducting nanowires with amyloid fibrils as templates [10]. PTAA is another polyelectrolyte, which is luminescent and amyloid fibrils can be coated with [11]. Amyloid fibrils represent a general class of nanomaterials which can be formed from many peptides and proteins [12]. An amyloid fibril has a pleated- $\beta$-sheet structure, formed under conditions that destabilize the native state of a protein, such as insulin [13]. Naked amyloid fibrils are electrically insulating and inert to light, as the native state of proteins. However, functionally decorated amyloid fibrils can be conducting [10] or luminescent [11], promising in a wide variety of applications, such as nanoelectronic devices, sensing, and drug delivery [12].

In this work, we report a comparative study on PEDOT:PSS electrochemical transistors with four different electrolytes, including conducting polymer PEDOT-S , luminescent polyelectrolyte PTAA and their corresponding insulin amyloid-fibril complexes. The absorbance and fluorescence of PTAA and its amyloid-fibril complex were studied to evaluate the interaction between the polyelectrolyte and the amyloid fibrils. The static and switching behaviors of all these electrochemical transistors were characterized and the discrimination of transistor behaviors is interpreted by electrochemical mechanism. The results show that the choice of gate electrolytes is crucial to the electrochemical transistors. The luminescent polymer PTAA implies a method to tune the color of PEDOT:PSS devices for electrochromic display. The demonstration of the polyelectrolytes and their amyloid-fibril complexes gating organic electrochemical transistors offers a possible integration for biological sensing and electrochromic display. 


\section{II.EXPERIMENTS}

The PEDOT:PSS electrochemical transistors were fabricated as [6], with a lateral structure schematically shown in Fig. 1(a). The PEDOT:PSS thin-film (blue, as received from AGFA-Geavert) coated on a polyester substrate (gray) was patterned into two shapes, the gate electrode separated from the source and drain electrodes that were connected via the transistor channel. Another layer of polyester (light green) with a window was aligned and laminated to the PEDOT:PSS patterns as a mask, which exposed the area for the electrolyte (red) to connect the gate and the channel. The channel area was around $250 \mu \mathrm{m} \times 500 \mu \mathrm{m}$. The conducting polymer PEDOT-S ( $1 \mathrm{mg} / \mathrm{mL}$ in distilled water, structure shown in Fig. 1b) was synthesized as [14]. The fluorescent polyelectrolyte PTAA ( $1 \mathrm{mg} / \mathrm{mL}$ in distilled water, structure shown in Fig. 1c) was reported elsewhere [15]. $320 \mu \mathrm{M}$ bovine insulin (Sigma-Aldrich) was dissolved in $25 \mathrm{mM} \mathrm{HCl}$, and then the solution was kept in an oven at $65^{\circ} \mathrm{C}$ over 6 hours to form insulin amyloid fibrils. The fibrillated insulin was decorated with PEDOT-S [10] and PTAA [11] as reported, respectively. The final concentrations of PEDOT-S and PTAA in polyelectrolyte/insulin fibril complex are $0.3 \mathrm{mg} / \mathrm{mL}$ and 0.1 $\mathrm{mg} / \mathrm{mL}$, respectively. For optical measurements, the PTAA solution was diluted to $0.1 \mathrm{mg} / \mathrm{mL}$ which was estimated to be the same as the concentration of PTAA in PTAA/insulin fibril complex. An ISA Jobin-Yvon spex FluoroMax-2 apparatus was used to record emission spectra for fluorescence measurements. The excitation wavelength was $450 \mathrm{~nm}$. For absorption measurements, a Lambda 950 spectrophotometer was used. A droplet $(10 \mu \mathrm{L})$ of the gate electrolytes was put on the device area which was defined by the laminated mask, connecting the gate and the channel. The electrical measurements were performed in an ambient atmosphere with a probe station and a Keithley 4200 SCS.

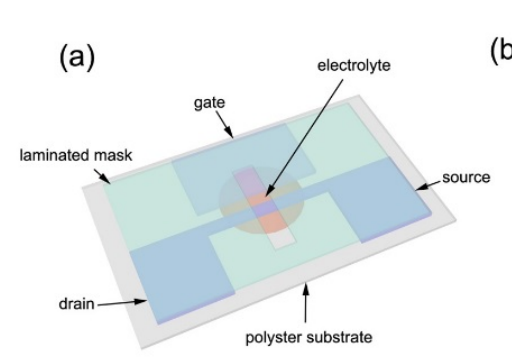

(c)
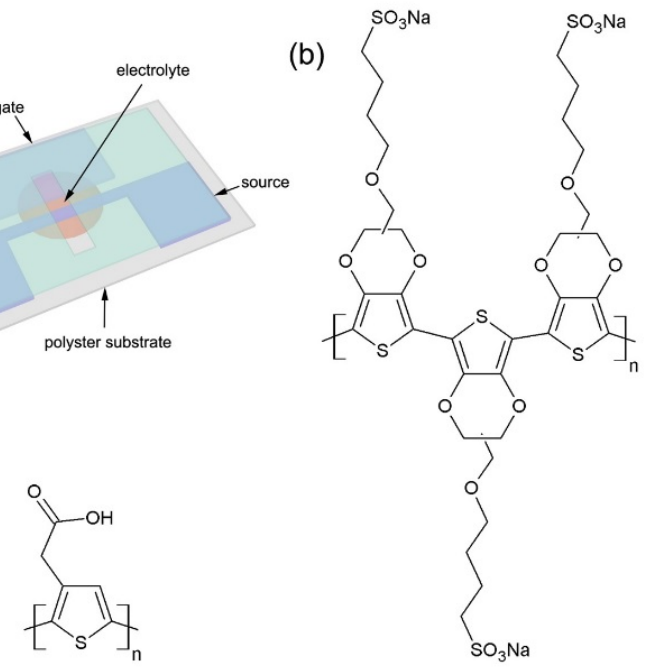

Fig. 1. (a) Schematic of a PEDOT:PSS electrochemical transistor with lateral structure. (b) Molecular structure of conducting polyelectrolyte PEDOT-S. (c) Molecular structure of fluorescent polyelectrolyte PTAA.

\section{RESULTS AND DISCUSSION}

\section{A. Optical characteristics}

The optical properties measured by absorption and fluorescence of PTAA and PTAA/amyloid fibrils complex are presented in Fig. 2. Here PEDOT-S was also characterized as reference, but it didn't give any spectrum of absorption and emission, due to its optical inertness. The maximum absorbance of PTAA is at $466 \mathrm{~nm}$ and the PTAA/amyloid fibrils complex slightly red-shifts to $468 \mathrm{~nm}$, shown in Fig. 2a. For the emission spectra, the maximum fluorescence intensity of PTAA is found at $578 \mathrm{~nm}$, while that of PTAA/amyloid fibrils complex is at $580 \mathrm{~nm}$, where a slight red-shift also appears. This red-shift is caused by molecular aggregation that is promoted by interchain backbone interactions [16]. Besides red-shift, there is an enhancement in fluorescence emission for PTAA/amyloid fibrils, consistent with previous results [16]. It is explained by that the interaction with fibrillar insulin gives an improved quantum yield [17]. However, the spectral discrimination is slight, because PTAA is a monomer-based polymer electrolyte with low degree of side-chain substitution along the polymer backbone.
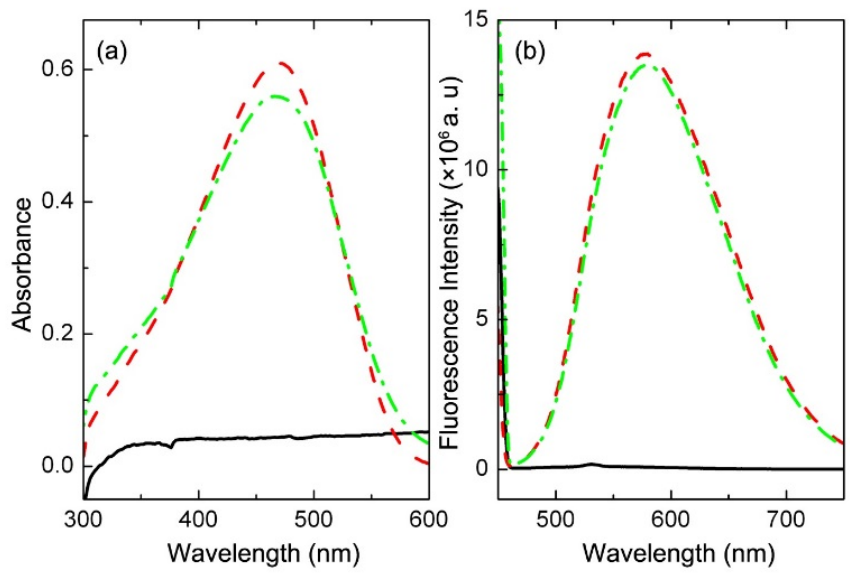

Fig. 2. (a) Absorbance and (b) fluorescence emission spectra of PEDOT-S (solid line), PTAA (dash-dot line), and PTAA/amyloid fibrils complex (dash line), respectively.

\section{B. DC characteristics}

The output $\left(I_{D}-V_{D}\right)$ characteristics of electrochemical transistors gated by four different electrolytes are presented in Fig. 3. During $I_{D}-V_{D}$ measurements, the drain voltage swept from 0 to $-1.5 \mathrm{~V}$ with the source grounded, while the gate voltage was kept at 0 to $1 \mathrm{~V}$ with a step of $0.2 \mathrm{~V}$. The negative gate voltage was not applied to avoid over oxidization which can lead to an irreversible damage to the transistors. Applying a positive gate voltage, the cations in electrolytes were driven to the channel and assisted in the reduction of PEDOT ${ }^{+}$, resulting in modulation of channel conductivity. This transistor behavior is quite similar to p-type silicon junction gate field effect transistors (JFETs) that need a positive gate voltage to pinch-off the channel. To interpret this kind of transistors in term of electrochemistry, a three-electrode electrochemical cell is a comparable analog. The drain and source can be known as 

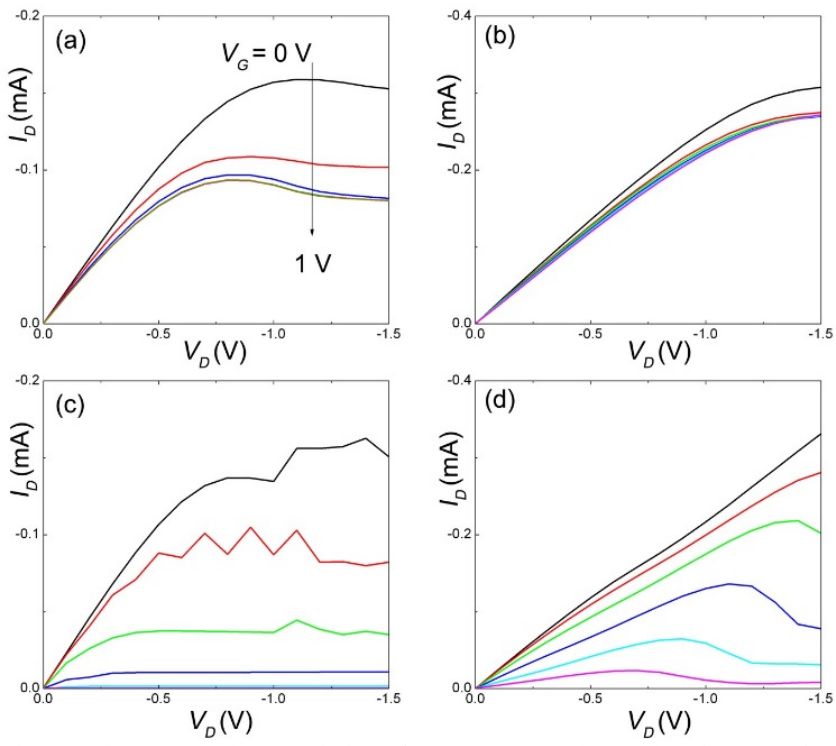

Fig. 3. The output characteristics of PEDOT:PSS electrochemical transistors with different electrolytes, including (a) PEDOT-S/amyloid fibrils complex, (b) PEDOT-S, (c) PTAA/amyloid fibrils complex and (d) PTAA.

the working electrode (WE) and the counter electrode (CE), while the gate is treated as the reference electrode (RE). Depending on the potential difference between WE and RE, an electrochemical charge transfer will take place and the PEDOT in the channel will be reduced or oxidized. The faradic current between WE and RE used to be low, compared with the channel current between WE and CE. Hence, the current at WE (drain current) can be modulated by the potential difference between WE and RE. As shown in Fig.3, all of the transistors exhibit drain current modulation by gate bias. However, the current modulation in the transistors gated by PTAA and PTAA/amyloid fibrils complex is much stronger than those gated by PEDOT-S, while the transistor with PEDOT-S/amyloid fibrils complex is in the middle. This indicates that PTAA and PTAA/amyloid fibrils complex are more efficient to reduce the PEDOT in the channel as gate electrolytes. Moreover, the transistors using PTAA and PTAA/amyloid fibrils complex exhibit better on/off ratios than other devices. Except PTAA, all other transistors show normal saturation behaviors, which are understood as that the concentration of the electrolytes was depleted closed to the channel surface. The depletion of electrolytes at the interface could even lead to a current drop after the drain voltage reached the reduction potential (Fig. 3a, c). The depletion of electrolytes is probably caused by the consumption of cations in the reduction shown in (1), as well as the loss of cations that penetrate into the channel. A current oscillation could also appear (Fig. 3c), which we have previously proposed an RLC equivalent circuit model to explain [18]. Nevertheless, another tentative explanation from an electrochemical point of view, which is still under development, follows the overpotential from the electrolyte diffusion and electron injection.

\section{Transient characteristics}

To further understand the electrochemical kinetic in the
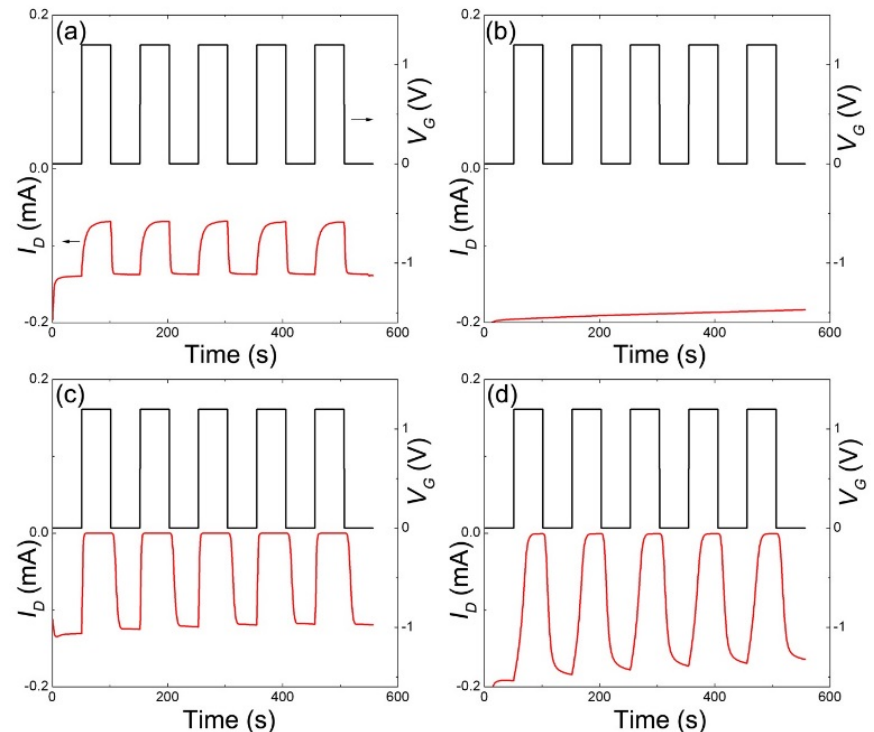

Fig. 4. The transient response of PEDOT:PSS electrochemical transistors with different electrolytes, including (a) PEDOT-S/amyloid fibrils complex, (b) PEDOT-S, (c) PTAA/amyloid fibrils complex, and (d) PTAA.

transistor operation, we measured the transient response of all the electrochemical transistors, presented in Fig. 4. The input applied at the gate was five $1.2-\mathrm{V}$ pulses with duration of $50 \mathrm{~s}$ over $550 \mathrm{~s}$ and the drain current was recorded as the output. As shown in Fig. 4, except the transistor with PEDOT-S as the gate electrolyte, all the others exhibit evident switching behaviors. The drain current of the transistor with PEDOT-S kept decreasing but could not be pinched-off. This is probably due to that the reduction of PEDOT takes place in the electrolyte rather than in the channel. Plus, the highly conductive PEDOT-S leads to a large leakage current at the gate.

Moreover, we analyzed the data and found that the on/off ratios (Fig. 5a) are 2 (PEDOT-S/Amyloid fibrils complex), 200 (PTAA), and 1500 (PTAA/Amyloid fibrils complex), respectively. The polyelectrolyte/amyloid fibrils complex presents an improved on/off ratio both for PEDOT-S and PTAA, compared with their pristine electrolytes. The binding of charged polymer and amyloid fibrils suppresses electron transport through the conducting electrolyte and makes the complex behave like ions.

From the data in Fig. 4, we also estimated the switching speed of all the transistors, presented in Fig. 5b. The switching-off time $\tau_{\text {off }}$ is defined as time between $100 \%$ to $10 \%$ of drain current $\left(I_{D}\right)$, while the switching-on time $\tau_{o n}$ is defined as time between 0 to $90 \%$ of $I_{D}$. The devices with PEDOT-S/amyloid fibrils and PTAA exhibited shorter $\tau_{\text {on }}$ than $\tau_{\text {off }}$, which means that is easier to recover the conductivity from the reduction of the PEDOT. In addition, a compromise to ion mobility caused by large polymer molecules carrying charges is considered to explain the slow operation speed. However, the PTAA/amyloid fibrils complex presented improved switching behaviors both in $\tau_{\text {off }}$ and $\tau_{\text {on }}$, especially for $\tau_{\text {off }}$. This may be understood by that the aggregation of PTAA and amyloid fibrils releases some space that makes the ion transport easier. To improve the speed of electrochemical transistors, another 
approach for consideration is to use a vertical structure instead of the lateral structure used here [6].

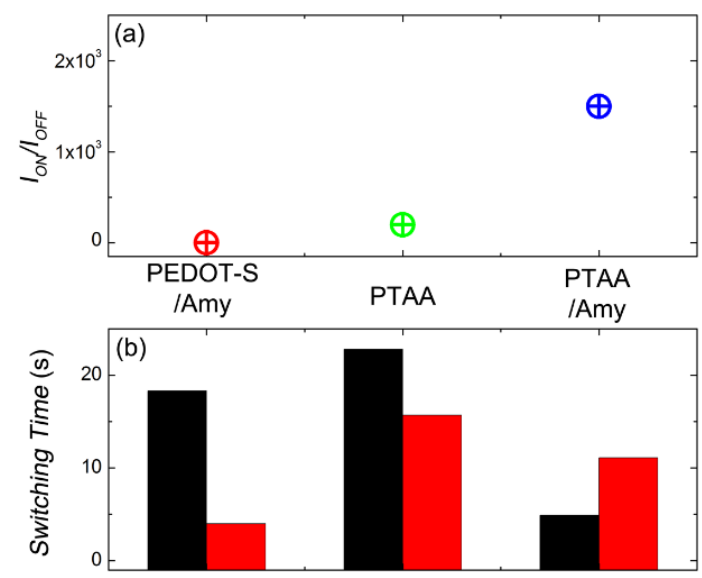

Fig. 5. (a) The on/off ratio of PEDOT:PSS electrochemical transistors with different electrolytes. (b) The switching time $\tau_{\text {off }}$ (left column) and $\tau_{\text {on }}$ (right column) of PEDOT:PSS electrochemical transistors with different electrolytes.

\section{CONCLUSION}

In summary, we have shown that conjugated polyelectrolytes (PTAA and PEDOT-S) and their complexes with biomolecules (Insulin amyloid fibrils) can gate the PEDOT:PSS electrochemical transistors, enabling the devices with possible new functionalities. The absorbance and fluorescent emission of PTAA and its biomolecular complex indicate the interaction between the polyelectrolyte and amyloid fibrils. The comparative study of transistor behaviors among six different gate electrolytes shows that the gate electrolyte is very important to the transistor performance. The incorporation of biomolecules into conducting polyelectrolytes and its integration with the electrical functionality of transistors pave a new way for real-time, reagent-free biosensing [19]. Furthermore, the fluorescent polyelectrolyte provides solution to adjust the colors in PEDOT:PSS devices for electrochromic display, which are used to be blue and white only [20].

\section{REFERENCES}

[1] H. Sirringhaus, T. Kawase, R. H. Friend, T. Shimoda, M. Inbasekaran, W. Wu, and E. P. Woo, "High-Resolution Inkjet Printing of All-Polymer Transistor Circuits,” Science, vol. 290, no. 5499, pp. 2123-2126, Dec. 2000.

[2] M. Hamedi, R. Forchheimer, and O. Inganäs, "Towards woven logic from organic electronic fibres," Nature Mater., vol. 6, no. 5, pp. 357-362, May 2007.

[3] H. Klauk, "Organic thin-film transistors," Chemical Society Reviews, vol. 39, no. 7, pp. 2643-2666, June 2010.

[4] J. W. Thackeray, H. S. White, and M. S. Wrighton, "Poly(3-methylthiophene)-coated electrodes: optical and electrical properties as a function of redox potential and amplification of electrical and chemical signals using poly(3-methylthiophene)-based microelectrochemical transistors," J. Phys. Chem., vol. 89, no. 23, pp. 5133-5140, Nov. 1985

[5] D. Nilsson, M. X. Chen, T. Kugler, T. Remonen, M. Armgarth, and M. Berggren, "Bi-stable and dynamic modulation in electrochemical organic transistors,” Adv. Mater., vol. 14, no. 1, pp. 51-54, Jan. 2002.
[6] D. Nilsson, N. Robinson, M. Berggren, and R. Forchheimer,

"Electrochemical logic circuits," Adv. Mater., vol. 17, no. 3, pp. 353-358, Feb. 2005.

[7] R. Mannerbro, M. Ranlöf, N. Robinson and R. Forchheimer, "Inkjet printed electrochemical organic electronics," Synth. Met., vol. 158, no. 13, pp. 556-560, Aug. 2008.

[8] Z. -T. Zhu, J. T. Mabeck, C. Zhu, N. C. Cady, C. A. Batt, and G. G. Malliaras, "A simple poly(3,4-ethylene dioxythiophene)/poly(styrene sulfonic acid) transistor for glucose sensing at neutral $\mathrm{pH}$," Chem. Comm., vol. 13, pp. 1556-1557, Jul. 2004.

[9] P. Andersson, D. Nilsson, P. -O. Svensson, M. Chen, A. Malmstrom, T. Remonen, T. Kugler, and M. Berggren, "Active matrix displays based on all-organic electrochemical smart pixels printed on paper," Adv. Mater., vol. 14, no. 20, pp. 1460-1464, Oct. 2002.

[10] M. Hamedi, A. Herland, R. H. Karlsson, and O. Inganäs, "Electrochemical Devices Made from Conducting Nanowire Networks Self-Assembled from Amyloid Fibrils and Alkoxysulfonate PEDOT,” Nano Lett.,vol. 8, no. 6, pp. 1736-1740, May 2008.

[11] A. Herland, P. Björk, P. R. Hania, I. G. Scheblykin, and O. Inganäs, "Alignment of a Conjugated Polymer onto Amyloid-like Protein Fibrils," Small, vol. 3, no. 2, pp. 318-325, Feb. 2007.

[12] T. P. J. Knowles and M. J. Buehler, "Nanomechanics of functional and pathological amyloid materials," Nature Nanotech., vol. 6 pp. 469-479, Jul. 2011.

[13] M. Sunde, L. C. Serpell, M. Bartlam, P. E. Fraser, M. B. Pepys, and C. C. Blake, "Common core structure of amyloid fibrils by synchrotron X-ray diffraction,” J. Mol. Biol., vol. 273, no. 3, pp. 729-739, Oct. 1997.

[14] R. H. Karlsson, A. Herland, M. Hamedi, J. A. Wigenius, A. Åslund, X. Liu, M. Fahlman, O. Inganäs, and P. Konradsson, "Iron-Catalyzed Polymerization of Alkoxysulfonate-Functionalized 3,4-Ethylenedioxythiophene Gives Water-Soluble Poly(3,4-ethylenedioxythiophene) of High Conductivity," Chemistry of Materials, vol. 21, no. 9, pp. 1815-1821, Apr. 2009.

[15] L. Ding, M. Jonforsen, L. S. Roman, M. R. Andersson, and O. Inganäs, "Photovoltaic cells with a conjugated polyelectrolyte," Synth. Met., vol. 110, no. 2, pp. 133-140, Apr. 2000.

[16] A. Åslund, A. Herland, P. Hammarström, K. P. R. Nilsson, B. -H. Jonsson, O. Inganäs, and P. Konradsson, "Studies of luminescent conjugated polythiophene derivatives-Enhanced spectral discrimination of protein conformational states," Bioconjugate Chem., vol. 18, no. 6, pp. 1860-1868, Nov-Dec. 2007.

[17] K. P. R. Nilsson, A. Åslund, I. Berg, S. Nyström, P. Konradsson, A. Herland, O. Inganäs, F. Stabo-Eeg, M. Lindgrem, G. T. Westermark, L. Lannfelt, L. N. G. Nilsson, and P. Hammarström, "Imaging Distinct Conformational States of Amyloid- $\beta$ Fibrils in Alzheimer's Disease Using Novel Luminescent Probes,” ACS Chem. Biol., vol. 2, no. 8, pp. 553-560, Aug. 2007.

[18] D. Tu and R. Forchheimer, "Self-oscillation in electrochemical transistors: An RLC modeling approach,” Solid-State Electron., vol. 69, no. , pp. 7-10, Mar. 2012.

[19] J. A. Arter, D. K. Taggart, T. M. McIntire, R. M. Penner, and G. A. Weiss, "Virus-PEDOT Nanowires for Biosensing," Nano Lett., vol. 10, no. 12, pp. 4858-4862, Nov. 2010.

[20] E. Said, P. Andersson, I. Engquist, X. Crispin, and M. Berggren, "Electrochromic Display Cells Driven by an Electrolyte-Gated Organic Field-Effect Transistor,” Organic Electron., vol. 10, no. 6, pp. 1195-1199, Sep. 2009.

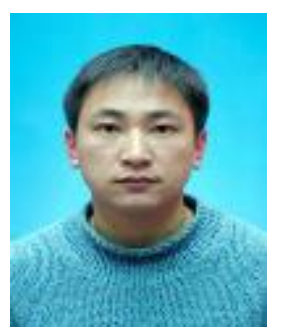

Deyu Tu received the B.S. degree in mechanism engineering and automatization from the Tsinghua University, Beijing, China, in 2003 and the Ph.D. degree in microelectronics and solid-state electronics from the Institute of Microelectronics, Chinese Academy of Sciences, Beijing, in 2008. From 2008 to 2010, he was a Postdoctoral Researcher at National Nanotechnology Laboratory of Consiglio Nazionale delle Ricerche-Istituto Nanoscienze, Lecce, Italy. He joined the Information 
Coding of Linköping University for the “OPEN” project in 2010. His research activities focus on the modeling and simulation of organic electronic devices and circuits, as well as the fabrication and characteristics.

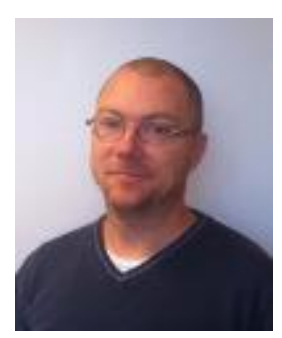

David Nilsson received the M.Sc. degree in physics at Linköping University in 2000. In 2005 he received his Ph.D. degree in Organic Electronics regarding work on organic electrochemical transistors.

Currently, he works as research scientist and project manager at the research institute Acreo $\mathrm{AB}$ within the department of printed electronics. His research interests focus on printed electronics based on electrochemical transistors and bioelectronics.

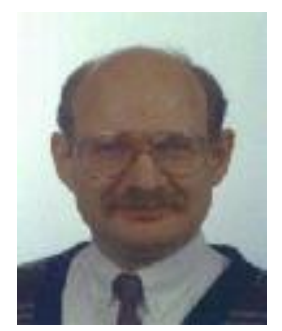

Robert Forchheimer was born in Gothenburg, Sweden in 1948. He received his Diploma degree in electrical engineering at the Royal Institute of Technology (KTH), Stockholm, Sweden in 1972 and Ph. D. in information theory at Linköping University, Linköping, Sweden in 1979.

He has held positions as Researcher and Lecturer at Linköping University, Visiting Scientist at Univ. of Southern California, USA and Visiting Professor at Universität Hannover, Germany. He is currently Professor at Linköping University where he heads a research group in Information Coding. His current interests are organic electronics, systems biology and optical networks. He has previously contributed to the fields of image and video coding, packet radio communication, smart optical sensors and optical computing.

Prof. Forchheimer was associate editor for IEEE Tr. Image Processing during 1996-1998. He has further been a member of Incentive Advisory Board and a member of the evaluation committee of the Swedish Board for Technical Development (NUTEK). In 2006 he became recipient of the Picture Coding Symposium (PCS) Award on the occasion of its 25th anniversary in recognition for his work in the picture coding field. 